\title{
On Polynomial Stability of Variational Nonautonomous Difference Equations in Banach Spaces
}

\author{
Mihail Megan, ${ }^{1,2}$ Traian Ceauşu, ${ }^{2}$ and Mihaela Aurelia Tomescu ${ }^{3}$ \\ ${ }^{1}$ Academy of Romanian Scientists, Independenţei 54, 050094 Bucharest, Romania \\ ${ }^{2}$ West University of Timişoara, Department of Mathematics, V. Pârvan Boulevard, No. 4, 300223 Timişoara, Romania \\ ${ }^{3}$ University of Petroşani, Department of Mathematics, University Street 20, 332006 Petroșani, Romania \\ Correspondence should be addressed to Mihaela Aurelia Tomescu; mtomescu@upet.ro
}

Received 4 December 2012; Accepted 5 February 2013

Academic Editor: Seenith Sivasundaram

Copyright (C) 2013 Mihail Megan et al. This is an open access article distributed under the Creative Commons Attribution License, which permits unrestricted use, distribution, and reproduction in any medium, provided the original work is properly cited.

Our goal in this paper is to give characterizations for some concepts of polynomial stability for variational nonautonomous difference equations. The obtained results can be considered generalizations for the case of variational nonautonomous difference equations of some theorems proved by Barbashin (1967), Datko (1973), and Lyapunov (1992), for evolution operators.

\section{Introduction}

In this paper we define and characterize two types of polynomial stability: (nonuniform) polynomial stability and strong polynomial stability for variational nonautonomous difference equations. These concepts are different from the concept of exponential stability studied for variational nonautonomous difference equations in [1], as shown in this paper.

In the case of evolution operators, the concept of nonuniform polynomial stability was studied by Barreira and Valls [2]. Moreover, characterizations for polynomial stability of evolution operators have been given in [3].

The variational nonautonomous difference equations considered in this paper generate discrete evolution cocycle over a discrete evolution semiflow. The concept of evolution cocycle was introduced by Megan and Stoica in [4].

We will consider the sets $\Delta=\left\{(m, n) \in \mathbb{N}^{2}\right.$, with $m \geq$ $n\}$ and $T=\left\{(m, n, p) \in \mathbb{N}^{3}\right.$, with $\left.m \geq n \geq p\right\}$, a metric space $(X, d)$ and $V$ a real or complex Banach space. The norm on $V$ and on $\mathscr{B}(V)$ (the Banach algebra of all bounded linear operators on $V$ ) will be denoted by $\|\cdot\|$.
Definition 1. A mapping $\varphi: \Delta \times X \rightarrow X$ is called a discrete evolution semiflow on $X$ if the following conditions hold:

$$
\begin{aligned}
& \left(s_{1}\right) \varphi(n, n, x)=x, \text { for all }(n, x) \in \mathbb{N} \times X ; \\
& \left(s_{2}\right) \varphi(m, n, \varphi(n, p, x))=\varphi(m, p, x), \text { for all }(m, n, p, x) \in \\
& \quad T \times X .
\end{aligned}
$$

Given a sequence $\left(A_{m}\right)_{m \in \mathbb{N}}$ with $A_{m}: X \rightarrow \mathscr{B}(V)$ and a discrete evolution semiflow $\varphi: \Delta \times X \rightarrow X$, we consider the problem of existence of a sequence $\left(v_{m}\right)_{m \in \mathbb{N}}$ with $v_{m}: \mathbb{N} \times$ $X \rightarrow X$ such that

$$
v_{m+1}(n, x)=A_{m}(\varphi(m, n, x)) v_{m}(n, x)
$$

for all $(m, n, x) \in \Delta \times X$. We will denote this problem by $(A, \varphi)$ and we say that $(A, \varphi)$ is a variational (nonautonomous) discrete-time system.

For $(m, n) \in \Delta$ we define the application $\Phi_{m}^{n}: X \rightarrow$ $\mathscr{B}(V)$ by

$$
\begin{aligned}
& \Phi_{m}^{n}(x) v \\
& = \begin{cases}A_{m-1}(\varphi(m-1, n, x)) \cdots A_{n+1}(\varphi(n+1, n, x)) A_{n}(x) v \\
v, & \text { if } m>n\end{cases} \\
& \text { if } m=n .
\end{aligned}
$$


Remark 2. From the definitions of $v_{m}$ and $\Phi_{m}^{n}$ it follows that

$\left(c_{1}\right) \Phi_{m}^{m}(x) v=v$, for all $(m, x, v) \in \mathbb{N} \times X \times V$;

$\left(c_{2}\right) \Phi_{m}^{p}(x)=\Phi_{m}^{n}(\varphi(n, p, x)) \Phi_{n}^{p}(x)$, for all $(m, n, p, x) \in$ $T \times X$

$\left(c_{3}\right) v_{m}(n, x)=\Phi_{m}^{n}(x) v_{n}(n, x)$, for all $(m, n, x) \in \Delta \times X$.

Definition 3. A mapping $\Phi: \Delta \times X \rightarrow \mathscr{B}(V)$ is called a discrete evolution cocycle over the discrete evolution semiflow $\varphi: \Delta \times X \rightarrow X$ if the following properties hold:

$\left(c_{1}\right) \Phi(n, n, x)=I$ (the identity operator on $V$ ), for all $(n, x) \in \mathbb{N} \times X$

$\left(c_{2}\right) \Phi(m, p, x)=\Phi(m, n,(\varphi(n, p, x)) \Phi(n, p, x)$, for all $(m, n, p, x) \in T \times X$.

If $\Phi$ is a discrete evolution cocycle over the discrete evolution semiflow $\varphi$, then the pair $S=(\Phi, \varphi)$ is called a discrete skewevolution semiflow on $X$.

Remark 4. From Remark 2 it results that the mapping

$$
\Phi: \Delta \times X \rightarrow \mathscr{B}(V), \quad \Phi(m, n, x) v=\Phi_{m}^{n}(x) v
$$

is a discrete evolution cocycle over discrete evolution semiflow $\varphi$.

\section{Polynomial Stability}

Let $(A, \varphi)$ be a discrete variational system associated with the discrete evolution semiflow $\varphi: \Delta \times X \rightarrow X$ and with the sequence of mappings $A=\left(A_{m}\right)$, where $A_{m}: X \rightarrow \mathscr{B}(V)$, for all $m \in \mathbb{N}$.

Definition 5. The system $(A, \varphi)$ is said to be

(i) exponentially stable (and denoted as e.s.) if there exist the constants $N \geq 1, \alpha>0$ and $\beta \geq 0$, such that

$$
e^{\alpha(m-n)}\left\|\Phi_{m}^{n}(x) v\right\| \leq N e^{\beta n}\|v\|
$$

for all $(m, n, x, v) \in \Delta \times X \times V$

(ii) polynomially stable (and denoted as p.s.) if there exist the constants $N \geq 1, \alpha>0$ and $\beta \geq 0$ such that

$$
(m+1)^{\alpha}\left\|\Phi_{m}^{n}(x) v\right\| \leq N(n+1)^{\alpha+\beta}\|v\|
$$

for all $(m, n, x, v) \in \Delta \times X \times V$.

Remark 6. The system $(A, \varphi)$ is

(i) exponentially stable if and only if there are $N \geq 1$, $\alpha>0$, and $\beta \geq 0$ with

$$
e^{\alpha(m-n)}\left\|\Phi_{m}^{p}(x) v\right\| \leq N e^{\beta n}\left\|\Phi_{n}^{p}(x) v\right\|
$$

for all $(m, n, p, x, v) \in T \times X \times V$; (ii) polynomially stable if and only if there exist $N \geq 1$, $\alpha>0$, and $\beta \geq 0$ with

$$
\begin{aligned}
& (m+1)^{\alpha}\left\|\Phi_{m}^{p}(x) v\right\| \leq N(n+1)^{\alpha+\beta}\left\|\Phi_{n}^{p}(x) v\right\| \\
& \text { for all }(m, n, p, x, v) \in T \times X \times V .
\end{aligned}
$$

The connection between the two concepts of stability defined previously is established in the following.

Remark 7. It is obvious that

$$
\text { e.s. } \Longrightarrow \text { p.s. }
$$

The following example shows that the converse implication is not valid.

Example 8. Let $\mathscr{C}=\mathscr{C}\left(\mathbb{R}_{+}, \mathbb{R}\right)$ be the metric space of all bounded continuous functions $x: \mathbb{R}_{+} \rightarrow \mathbb{R}$, with the topology of uniform convergence. $\mathscr{C}$ is metrizable with respect to the metric $d\left(x_{1}, x_{2}\right)=\sup _{t \in \mathbb{R}_{+}}\left|x_{1}(t)-x_{2}(t)\right|$. Let $f: \mathbb{R}_{+} \rightarrow(0, \infty)$ be a bounded decreasing function with the property that there exists $\lim _{t \rightarrow \infty} f(t)=l>0$. We denote by $X$ the closure in $\mathscr{C}$ of the set $\left\{f_{t}, t \in \mathbb{R}_{+}\right\}$, where $f_{t}(s)=f(t+s)$ for all $s \in \mathbb{R}_{+}$. The mapping $\varphi: \Delta \times X \rightarrow X$ defined by $\varphi(m, n, x)=x_{m-n}$ is a discrete evolution semiflow. Let us consider the Banach space $V$ and let the sequence of mappings $A_{m}: X \rightarrow \mathscr{B}(V)$, defined by

$$
A_{m}(x) v=\frac{u(m) x(\tau)}{u(m+1) x(\tau+1)} v
$$

for all $(m, x, v) \in \mathbb{N} \times X \times V$, where the sequence $u: \mathbb{N} \rightarrow \mathbb{R}$ is given by

$$
u(m)=(m+1)\left(m+1-m \cos \frac{m \pi}{2}\right)^{2} .
$$

Then

$$
\Phi_{m}^{n}(x) v=\frac{(n+1)(n+1-n \cos (n \pi / 2))^{2} x(\tau)}{(m+1)(m+1-m \cos (m \pi / 2))^{2} x(m-n+\tau)} v,
$$

and it results that

$$
\begin{aligned}
\left\|\Phi_{m}^{n}(x) v\right\| & \leq(2 n+1)^{2} \frac{(n+1) x(0)}{(m+1) l}\|v\| \\
& \leq N \frac{n+1}{m+1}(n+1)^{2}\|v\|
\end{aligned}
$$

for all $(m, n, x, v) \in \Delta \times X \times V$, where $N=4 x(0) / l$. Hence $(A, \varphi)$ is p.s. Assume by a contradiction that $(A, \varphi)$ is e.s. According to Definition 5, there are $N \geq 1, \alpha>0$, and $\beta \geq 0$ such that

$$
e^{\alpha(m-n)}\left\|\Phi_{m}^{n}(x) v\right\| \leq N e^{\beta n}\|v\|
$$

for all $(m, n, x, v) \in \Delta \times X \times V$. The previous inequality for the considered system becomes

$e^{\alpha(m-n)}$

$$
\times \frac{(n+1)(n+1-n \cos (n \pi / 2))^{2} x(\tau)}{(m+1)(m+1-m \cos (m \pi / 2))^{2} x(m-n+\tau)} \leq N e^{\beta n}
$$


for all $(m, n, x) \in \Delta \times X$. If we take $m=4 k^{2}+4$ and $n=4 k+2$, $k \in \mathbb{N}$, then

$$
\begin{aligned}
e^{4 \alpha k^{2}+k(\alpha-\beta-2)+2} \frac{(4 k+3)(8 k+5)^{2}}{4 k^{2}+5} \\
\times \frac{x(\tau)}{x\left(4 k^{2}-4 k+2+\tau\right)} \leq N e^{2 \beta} .
\end{aligned}
$$

Passing to the limit for $k \rightarrow \infty$ we obtain a contradiction. We have shown that $(A, \varphi)$ is not e.s.

Lemma 9. The system $(A, \varphi)$ is polynomially stable if and only if there are $N \geq 1$ and $0<c \leq d$ such that

$$
(m+1)^{c}\left\|\Phi_{m}^{n}(x) v\right\| \leq N(n+1)^{d}\|v\|
$$

for all $(m, n, x, v) \in \Delta \times X \times V$.

Proof. Necessity. If $(A, \varphi)$ is p.s., then there are $N \geq 1, \alpha>0$, and $\beta \geq 0$ such that

$$
(m+1)^{\alpha}\left\|\Phi_{m}^{n}(x) v\right\| \leq N(n+1)^{\alpha+\beta}\|v\|
$$

for all ( $m, n, x, v) \in \Delta \times X \times V$. Hence inequality (16) holds for $c=\alpha$ and $d=\alpha+\beta$.

Sufficiency. From the hypothesis it results that relation (5) of Definition 5 holds for $\alpha=c$ and $\beta=d-c$.

A necessary condition for the polynomial stability property is presented by the following theorem.

Theorem 10. If the system $(A, \varphi)$ is polynomially stable, then there are $D \geq 1, d>0, \omega>0$, and $\gamma \geq 0$ such that

$$
\sum_{k=n}^{\infty} \frac{1}{k+1}\left(\frac{k+1}{n+1}\right)^{d}\left\|\Phi_{k}^{n}(x) v\right\| \leq D(n+1)^{\gamma}\|v\|
$$

for all $(n, x, v) \in \mathbb{N} \times X \times V$ and

$$
\left\|\Phi_{m}^{n}(x) v\right\| \leq D(m+1)^{\omega}(n+1)^{\gamma-\omega}\|v\|
$$

for all $(m, n, x, v) \in \Delta \times X \times V$.

Proof. Let $N \geq 1, \alpha>0$, and $\beta \geq 0$ as in Definition 5. Then, for every $d \in(0, \alpha)$ we have that

$$
\begin{aligned}
& \sum_{k=n}^{\infty} \frac{1}{k+1}\left(\frac{k+1}{n+1}\right)^{d}\left\|\Phi_{k}^{n}(x) v\right\| \\
& \leq N(n+1)^{\alpha+\beta-d}\|v\| \sum_{k=n}^{\infty} \frac{1}{(k+1)^{\alpha+1-d}} \\
& =N\|v\|(n+1)^{\alpha+\beta-d}\left(\frac{1}{(n+1)^{\alpha+1-d}}+\sum_{k=n+1}^{\infty} \frac{1}{(k+1)^{\alpha+1-d}}\right)
\end{aligned}
$$

$$
\begin{aligned}
& \leq N\|v\|(n+1)^{\alpha+\beta-d}\left(\frac{1}{(n+1)^{\alpha+1-d}}+\frac{1}{\alpha-d} \frac{1}{(n+1)^{\alpha-d}}\right) \\
& \leq N\|v\|(n+1)^{\alpha+\beta-d}\left(1+\frac{1}{\alpha-d}\right) \frac{1}{(n+1)^{\alpha-d}} \\
& =\frac{N(1+\alpha-d)}{\alpha-d}\|v\|(n+1)^{\beta} \leq D\|v\|(n+1)^{\gamma}
\end{aligned}
$$

for all $(n, x, v) \in \mathbb{N} \times X \times V$, where $D=N(1+\alpha-d) /(\alpha-d)$ and $\gamma=\beta$. In addition

$$
\begin{aligned}
\left\|\Phi_{m}^{n}(x) v\right\| & \leq N \frac{(n+1)^{\alpha+\beta}}{(m+1)^{\alpha}}\|v\| \\
& =N\left(\frac{m+1}{n+1}\right)^{\omega}\left(\frac{n+1}{m+1}\right)^{\alpha+\omega}(n+1)^{\beta}\|v\| \\
& \leq D\left(\frac{m+1}{n+1}\right)^{\omega}(n+1)^{\gamma}\|v\|
\end{aligned}
$$

for all $(m, n, x, v) \in \Delta \times X \times V$.

Next, a sufficient condition for the polynomial stability property is presented by.

Theorem 11. If there are $D \geq 1$ and $d>\omega>\gamma \geq 0$ such that

$$
\sum_{k=n}^{\infty} \frac{1}{k+1}\left(\frac{k+1}{n+1}\right)^{d}\left\|\Phi_{k}^{n}(x) v\right\| \leq D(n+1)^{\gamma}\|v\|
$$

for all $(n, x, v) \in \mathbb{N} \times X \times V$ and

$$
\left\|\Phi_{m}^{n}(x) v\right\| \leq D(m+1)^{\omega}(n+1)^{\gamma-\omega}\|v\|
$$

for all $(m, n, x, v) \in \Delta \times X \times V$, then the system $(A, \varphi)$ is polynomially stable.

Proof. From the hypothesis it results that

$$
\left\|\Phi_{m}^{n}(x) v\right\| \leq D(m+1)^{\omega}(k+1)^{\gamma-\omega}\left\|\Phi_{k}^{n}(x) v\right\|
$$

for all $(m, k, n, x, v) \in T \times X \times V$. We suppose that $m \geq 2 n$ and we denote by $j=[\mathrm{m} / 2]$. Then

$$
\begin{aligned}
& \frac{m+2}{2} \frac{(m+1)^{d-\omega}}{(n+1)^{d-\omega+\gamma}}\left\|\Phi_{m}^{n}(x) v\right\| \\
& \leq \sum_{k=j}^{m} \frac{(m+1)^{d-\omega}}{(n+1)^{d-\omega+\gamma}}\left\|\Phi_{m}^{n}(x) v\right\| \\
& \leq D \sum_{k=j}^{m} \frac{(m+1)^{d-\omega}}{(n+1)^{d-\omega+\gamma}}\left(\frac{m+1}{k+1}\right)^{\omega}
\end{aligned}
$$




$$
\begin{aligned}
& \times(k+1)^{\gamma}\left\|\Phi_{k}^{n}(x) v\right\| \\
= & D \sum_{k=j}^{m} \frac{1}{k+1}\left(\frac{k+1}{n+1}\right)^{d}\left\|\Phi_{k}^{n}(x) v\right\| \\
& \times\left(\frac{m+1}{k+1}\right)^{d}\left(\frac{n+1}{k+1}\right)^{\omega-\gamma}(k+1) \\
\leq & D 4^{d}(m+1) \sum_{k=j}^{m} \frac{1}{k+1}\left(\frac{k+1}{n+1}\right)^{d}\left\|\Phi_{k}^{n}(x) v\right\| \\
\leq & D 4^{d}(m+1) \sum_{k=n}^{\infty} \frac{1}{k+1}\left(\frac{k+1}{n+1}\right)^{d}\left\|\Phi_{k}^{n}(x) v\right\| \\
\leq & D^{2} 4^{d}(m+1)(n+1)^{\gamma}\|v\| .
\end{aligned}
$$

Hence,

$$
\begin{aligned}
& \left(\frac{m+1}{n+1}\right)^{d-\omega}\left\|\Phi_{m}^{n}(x) v\right\| \\
& \leq D^{2} 4^{d} \frac{2(m+1)}{m+2}(n+1)^{2 \gamma}\|v\| \\
& \leq D^{2} 2^{2 d+1}(n+1)^{2 \gamma}\|v\| \\
& \quad=N(n+1)^{2 \gamma}\|v\|
\end{aligned}
$$

for all $(x, v) \in X \times V$, where $N=D^{2} 2^{2 d+1}$. If $n \leq m<2 n$, then

$$
\begin{aligned}
& \left(\frac{m+1}{n+1}\right)^{d-\omega}\left\|\Phi_{m}^{n}(x) v\right\| \\
& \quad \leq D\left(\frac{m+1}{n+1}\right)^{d-\omega}\left(\frac{m+1}{n+1}\right)^{\omega}(n+1)^{\gamma}\|v\| \\
& \quad \leq D 2^{d}(n+1)^{\gamma}\|v\| \leq N(n+1)^{2 \gamma}\|v\|
\end{aligned}
$$

for all $(x, v) \in X \times V$ thus we have proved that $(A, \varphi)$ is p.s.

As a generalization of a theorem of Barbashin [5], we give the following characterization of the polynomial stability property.

Theorem 12. The system $(A, \varphi)$ is polynomially stable if and only if there are $B \geq 1$ and $a>b \geq 0$ such that

$$
\sum_{k=n}^{m} \frac{1}{k+1}\left(\frac{m+1}{k+1}\right)^{a}\left\|\Phi_{m}^{k}(x) v\right\| \leq B(m+1)^{b}\|v\|
$$

for all $(m, n, x, v) \in \Delta \times X \times V$.
Proof. Necessity. Let $N \geq 1, \alpha>0$, and $\beta \geq 0$ as in Definition 5. Then, for every $a>0$ with $0 \leq \beta<a<\alpha+\beta<$ $a+1$ we have that

$$
\begin{aligned}
& \sum_{k=n}^{m} \frac{1}{k+1}\left(\frac{m+1}{k+1}\right)^{a}\left\|\Phi_{m}^{k}(x) v\right\| \\
& \leq N\|v\| \sum_{k=n}^{m} \frac{1}{k+1}\left(\frac{m+1}{k+1}\right)^{a} \frac{(k+1)^{\alpha+\beta}}{(m+1)^{\alpha}} \\
& \quad N\|v\|(m+1)^{a-\alpha} \sum_{k=n}^{m} \frac{1}{(k+1)^{a+1-\alpha-\beta}} \\
& \leq N\|v\|(m+1)^{a-\alpha} \frac{1}{\alpha+\beta-a}(m+1)^{\alpha+\beta-a} \\
& \leq D(m+1)^{b}\|v\|
\end{aligned}
$$

for all $(m, n, x, v) \in \Delta \times X \times V$, where $D=N /(\alpha+\beta-a)$ and $b=\beta$.

Sufficiency. From the hypothesis we have

$$
\frac{1}{n+1}\left(\frac{m+1}{n+1}\right)^{a}\left\|\Phi_{m}^{n}(x) v\right\| \leq B(m+1)^{b}\|v\|
$$

for all $(m, n, x, v) \in \Delta \times X \times V$. Hence

$$
(m+1)^{a-b}\left\|\Phi_{m}^{n}(x) v\right\| \leq B(n+1)^{a+1}\|v\|,
$$

and relation (16) from Lemma 9 holds for $0<c=a-b \leq a<$ $a+1=d$.

Definition 13. An application $L: \Delta \times X \times V \rightarrow \mathbb{R}_{+}$is called a Lyapunov polynomial stability function for the system $(A, \varphi)$ if there exists $l>0$ such that

$$
L(m, p, x, v)+\sum_{k=n}^{m-1} \frac{1}{k+1}\left(\frac{k+1}{n+1}\right)^{l}\left\|\Phi_{k}^{p}(x) v\right\| \leq L(n, p, x, v)
$$

for all $(m, n, p, x, v) \in T \times X \times V$, with $m>n$.

The constant $l>0$ is called the order of the Lyapunov function $L$.

Theorem 14. If the system $(A, \varphi)$ is polynomially stable, then there are a Lyapunov polynomial stability function for the system $(A, \varphi)$ and constants $K \geq 1, v>0$ and $\delta \geq 0$ such that

$$
\begin{gathered}
L(m, n, x, v) \leq K(n+1)^{\delta}\|v\|, \\
\left\|\Phi_{m}^{n}(x) v\right\| \leq K(m+1)^{v}(n+1)^{\delta-v}\|v\|
\end{gathered}
$$

for all $(m, n, x, v) \in \Delta \times X \times V$.

Proof. From Theorem 10 we have that there are $D \geq 1, d>0$, $\omega>0$, and $\gamma \geq 0$ such that

$$
\begin{gathered}
\sum_{k=n}^{\infty} \frac{1}{k+1}\left(\frac{k+1}{n+1}\right)^{d}\left\|\Phi_{k}^{n}(x) v\right\| \leq D(n+1)^{\gamma}\|v\|, \\
\left\|\Phi_{m}^{n}(x) v\right\| \leq D(m+1)^{\omega}(n+1)^{\gamma-\omega}\|v\|
\end{gathered}
$$


for all $(m, n, x, v) \in \Delta \times X \times V$. We define the application $L: \Delta \times X \times V \rightarrow \mathbb{R}_{+}$by

$$
L(m, p, x, v)=\sum_{k=m}^{\infty} \frac{1}{k+1}\left(\frac{k+1}{m+1}\right)^{d}\left\|\Phi_{k}^{p}(x) v\right\|
$$

for all $(m, p, x, v) \in \Delta \times X \times V$. Then, for all $(m, n, p, \mathrm{x}, v) \in$ $T \times X \times V$, with $m>n$ we have

$$
\begin{gathered}
L(m, p, x, v)+\sum_{k=n}^{m-1} \frac{1}{k+1}\left(\frac{k+1}{n+1}\right)^{d}\left\|\Phi_{k}^{p}(x) v\right\| \\
=\sum_{k=m}^{\infty} \frac{1}{k+1}\left(\frac{k+1}{m+1}\right)^{d}\left\|\Phi_{k}^{p}(x) v\right\| \\
\quad+\sum_{k=n}^{m-1} \frac{1}{k+1}\left(\frac{k+1}{n+1}\right)^{d}\left\|\Phi_{k}^{p}(x) v\right\| \\
\leq \sum_{k=n}^{\infty} \frac{1}{k+1}\left(\frac{k+1}{n+1}\right)^{d}\left\|\Phi_{k}^{p}(x) v\right\| \\
=L(n, p, x, v) .
\end{gathered}
$$

In addition, from Theorem 10 we have that

$$
\begin{aligned}
L(m, n, x, v) & =\sum_{k=m}^{\infty} \frac{1}{k+1}\left(\frac{k+1}{n+1}\right)^{d}\left\|\Phi_{k}^{n}(x) v\right\| \\
& \leq \sum_{k=n}^{\infty} \frac{1}{k+1}\left(\frac{k+1}{n+1}\right)^{d}\left\|\Phi_{k}^{n}(x) v\right\| \\
& \leq D(n+1)^{\gamma}\|v\|, \\
\left\|\Phi_{m}^{n}(x) v\right\| & \leq D(m+1)^{\omega}(n+1)^{\gamma-\omega}\|v\| .
\end{aligned}
$$

Consequently, relations (33) are satisfied for $K=D, \delta=\gamma$ and $v=\omega$.

Theorem 15. If there exist a Lyapunov polynomial stability function with the order $l>0$ for the system $(A, \varphi)$ and the constants $K \geq 1, v>0$, and $\delta \geq 0$ with $l>v>\delta \geq 0$ such that

$$
\begin{gathered}
L(m, n, x, v) \leq K(n+1)^{\delta}\|v\|, \\
\left\|\Phi_{m}^{n}(x) v\right\| \leq K(m+1)^{v}(n+1)^{\delta-v}\|v\|
\end{gathered}
$$

for all $(m, n, x, v) \in \Delta \times X \times V$, then the system $(A, \varphi)$ is polynomially stable.

Proof. From the hypothesis and Definition 13 we have that

$$
\begin{aligned}
& \sum_{k=n}^{m-1} \frac{1}{k+1}\left(\frac{k+1}{n+1}\right)^{l}\left\|\Phi_{k}^{n}(x) v\right\| \\
& \quad \leq L(n, n, x, v)-L(m, n, x, v) \\
& \quad \leq L(n, n, x, v) \leq K(n+1)^{\delta}\|v\|
\end{aligned}
$$

for all $(m, n, x, v) \in \Delta \times X \times V$, with $m>n$. Passing to the limit for $m \rightarrow \infty$ we obtain that

$$
\sum_{k=n}^{\infty} \frac{1}{k+1}\left(\frac{k+1}{n+1}\right)^{l}\left\|\Phi_{k}^{n}(x) v\right\| \leq K(n+1)^{\delta}\|v\|
$$

for all $(n, x, v) \in \mathbb{N} \times X \times V$. Now, from Theorem 11 the conclusion follows.

\section{Strong Polynomial Stability}

Definition 16. The system $(A, \varphi)$ is said to be strongly polynomially stable (and denoted as s.p.s.) if there are three constants $N \geq 1$ and $\alpha>\beta \geq 0$ such that

$$
(m+1)^{\alpha}\left\|\Phi_{m}^{n}(x) v\right\| \leq N(n+1)^{\alpha+\beta}\|v\|
$$

for all $(m, n, x, v) \in \Delta \times X \times V$.

Remark 17. It is easy to see that $(A, \varphi)$ is strongly polynomially stable if and only if there are $N \geq 1$ and $\alpha>\beta \geq 0$ with

$$
(m+1)^{\alpha}\left\|\Phi_{m}^{p}(x) v\right\| \leq N(n+1)^{\alpha+\beta}\left\|\Phi_{n}^{p}(x) v\right\|
$$

for all $(m, n, p, x, v) \in T \times X \times V$.

Remark 18. It is obvious that

$$
\text { s.p.s. } \Longrightarrow \text { p.s. }
$$

The following example shows that the converse implication is not valid.

Example 19. Let $(X, d)$ be the metric space, let $V$ be a Banach space, and let $\varphi$ be the evolution semiflow given as in Example 8. We define the sequence of mappings $A_{m}: X \rightarrow$ $\mathscr{B}(\mathbb{R})$ by

$$
A_{m}(x) v=\frac{u(m) x(\tau)}{u(m+1) x(\tau+1)} v
$$

for all $(m, x, v) \in \mathbb{N} \times X \times V$, where the sequence $u: \mathbb{N} \rightarrow \mathbb{R}$ is given by

$$
u(m)=(m+1)\left(m+1-m \cos \frac{m \pi}{2}\right) .
$$

Then

$$
\Phi_{m}^{n}(x) v=\frac{(n+1)(n+1-n \cos (n \pi / 2)) x(\tau)}{(m+1)(m+1-m \cos (m \pi / 2)) x(m-n+\tau)} v,
$$

and it follows that

$$
\begin{aligned}
\left\|\Phi_{m}^{n}(x) v\right\| & \leq(2 n+1) \frac{(n+1) x(0)}{(m+1) l}\|v\| \\
& \leq N \frac{n+1}{m+1}(n+1)\|v\|
\end{aligned}
$$

for all $(m, n, x, v) \in \Delta \times X \times V$, where $N=2 x(0) / l$. Hence we have proved that $(A, \varphi)$ is p.s. Let us suppose now that the 
system $(A, \varphi)$ is s.p.s. According to Definition 16, there exist $N \geq 1$ and $\alpha>\beta \geq 0$ such that

$$
\begin{aligned}
& \frac{(n+1)(n+1-n \cos (n \pi / 2)) x(\tau)}{(m+1)(m+1-m \cos (m \pi / 2)) x(m-n+\tau)} \\
& \leq N\left(\frac{n+1}{m+1}\right)^{\alpha}(n+1)^{\beta}
\end{aligned}
$$

for all $(m, \mathrm{n}, x) \in \Delta \times X$. If we take $n=4 k+2$ and $m=4 k^{2}+4$, $k \in \mathbb{N}$, we have that

$$
\left(\frac{4 k+3}{4 k^{2}+5}\right)^{1-\alpha} \frac{8 k+5}{4 k+3} \frac{x(\tau)}{x\left(4 k^{2}-4 k+2+\tau\right)} \leq N(4 k+3)^{\beta-1} \text {. }
$$

It follows that $\alpha \leq 1, \beta \geq 1$, and $N \geq 2$ which implies that $0<\alpha \leq 1 \leq \beta$, contradicting the fact that $\alpha>\beta \geq 0$. This proves that $(A, \varphi)$ is not s.p.s.

Lemma 20. The system $(A, \varphi)$ is strongly polynomially stable if and only if there are $N \geq 1$ and $0<c \leq d<2 c$ such that

$$
(m+1)^{c}\left\|\Phi_{m}^{n}(x) v\right\| \leq N(n+1)^{d}\|v\|
$$

for all $(m, n, x, v) \in \Delta \times X \times V$.

Proof. It is similar to the proof of Lemma 9 with the condition $0<\alpha=c \leq d=\alpha+\beta<2 \alpha=2 c$ in the case of the necessity and with $0 \leq \beta=d-c<c=\alpha$ for the sufficiency.

Theorem 21. If the system $(A, \varphi)$ is strongly polynomially stable, then there are $D \geq 1, d>0, \omega>0$, and $\gamma \geq 0$ such that

$$
\sum_{k=n}^{\infty} \frac{1}{k+1}\left(\frac{k+1}{n+1}\right)^{d}\left\|\Phi_{k}^{n}(x) v\right\| \leq D(n+1)^{\gamma}\|v\|
$$

for all $(n, x, v) \in \mathbb{N} \times X \times V$,

$$
\left\|\Phi_{m}^{n}(x) v\right\| \leq D(m+1)^{\omega}(n+1)^{\gamma-\omega}\|v\|
$$

for all $(m, n, x, v) \in \Delta \times X \times V$.

Proof. It results from the proof of Theorem 10 with $0 \leq \beta<$ $d<\alpha$ for the first inequality and with $0 \leq \beta=\gamma<\omega<d<\alpha$ for the second inequality.

Theorem 22. If there are $D \geq 1$ and $d>\omega>\gamma \geq 0$ with $2 \gamma+\omega<d$ such that

$$
\sum_{k=n}^{\infty} \frac{1}{k+1}\left(\frac{k+1}{n+1}\right)^{d}\left\|\Phi_{k}^{n}(x) v\right\| \leq D(n+1)^{\gamma}\|v\|
$$

for all $(n, x, v) \in \mathbb{N} \times X \times V$ and

$$
\left\|\Phi_{m}^{n}(x) v\right\| \leq D(m+1)^{\omega}(n+1)^{\gamma-\omega}\|v\|
$$

for all $(m, n, x, v) \in \Delta \times X \times V$, then the system $(A, \varphi)$ is strongly polynomially stable.

Proof. It is analogous to the proof of Theorem 11.

Next, we present a generalization of a theorem due to Lyapunov for the case of strong polynomial stability of discrete variational systems.

Theorem 23. If the system $(A, \varphi)$ is strongly polynomially stable, then there exist a Lyapunov polynomial stability function for the system $(A, \varphi)$ and constants $K \geq 1$ and $0 \leq \delta<v$ such that

$$
\begin{gathered}
L(m, n, x, v) \leq K(n+1)^{\delta}\|v\|, \\
\left\|\Phi_{m}^{n}(x) v\right\| \leq K(m+1)^{v}(n+1)^{\delta-v}\|v\|
\end{gathered}
$$

for all $(m, n, x, v) \in \Delta \times X \times V$.

Proof. Using the technique from the proof of Theorem 14 for $0 \leq \gamma<\omega<d$ we obtain the conclusion.

Theorem 24. If there are a Lyapunov polynomial stability function with the order $l>0$ for the system $(A, \varphi)$ and the constants $K \geq 1, v>0$ and $\delta \geq 0$ with $l>v>\delta \geq 0$ and $2 \delta+v<l$ such that:

$$
\begin{gathered}
L(m, n, x, v) \leq K(n+1)^{\delta}\|v\|, \\
\left\|\Phi_{m}^{n}(x) v\right\| \leq K(m+1)^{v}(n+1)^{\delta-v}\|v\|
\end{gathered}
$$

for all $(m, n, x, v) \in \Delta \times X \times V$, then the system $(A, \varphi)$ is strongly polynomially stable.

Proof. It is the same as the proof of Theorem 15.

Remark 25. If the system $(A, \varphi)$ is strongly polynomially stable, then there are $B \geq 1$ and $a>b \geq 0$ such that

$$
\sum_{k=n}^{m} \frac{1}{k+1}\left(\frac{m+1}{k+1}\right)^{a}\left\|\Phi_{m}^{k}(x) v\right\| \leq B(m+1)^{b}\|v\|
$$

for all $(m, n, x, v) \in \Delta \times X \times V$.

Remark 26. If there are $B \geq 1, a>0$, and $b \geq 0$, with $a>$ $2 b+1$ such that

$$
\sum_{k=n}^{m} \frac{1}{k+1}\left(\frac{m+1}{k+1}\right)^{a}\left\|\Phi_{m}^{k}(x) v\right\| \leq B(m+1)^{b}\|v\|
$$

for all $(m, n, x, v) \in \Delta \times X \times V$, then the system $(A, \varphi)$ is strongly polynomially stable.

\section{References}

[1] M. Megan, T. Ceauşu, and M. A. Tomescu, "On exponential stability of variational nonautonomous difference equations in Banach spaces," Annals of the Academy of Romanian Scientists, vol. 4, no. 1, pp. 20-31, 2012. 
[2] L. Barreira and C. Valls, "Polynomial growth rates," Nonlinear Analysis: Theory, Methods \& Applications, vol. 71, no. 11, pp. 5208-5219, 2009.

[3] M. Megan, T. Ceauşu, and M. L. Ramneanţu, "Polynomial stability of evolution operators in Banach spaces," Opuscula Mathematica, vol. 31, no. 2, pp. 279-288, 2011.

[4] M. Megan and C. Stoica, "Discrete asymptotic behaviors for skew-evolution semi ows on Banach spaces," Carpathian Journal of Mathematics, vol. 24, pp. 348-355, 2008.

[5] E. A. Barbashin, Introduction in Stability Theory, Nauka, Moscow, Russia 1967. 


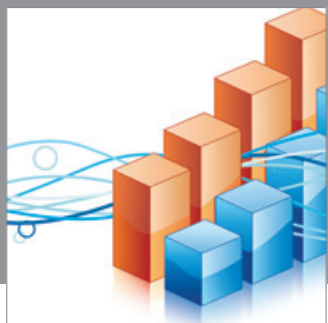

Advances in

Operations Research

mansans

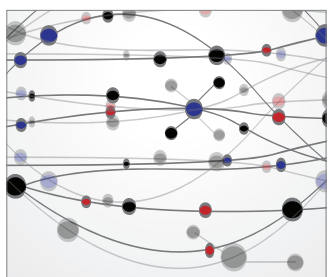

The Scientific World Journal
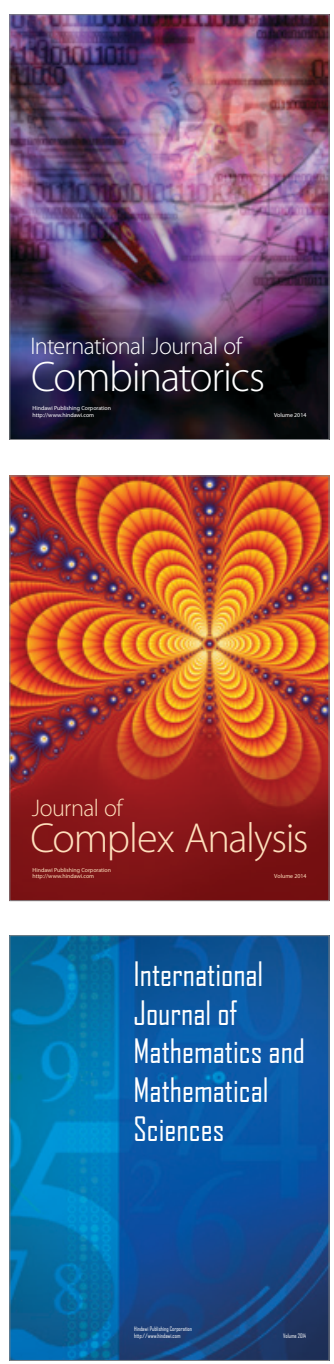
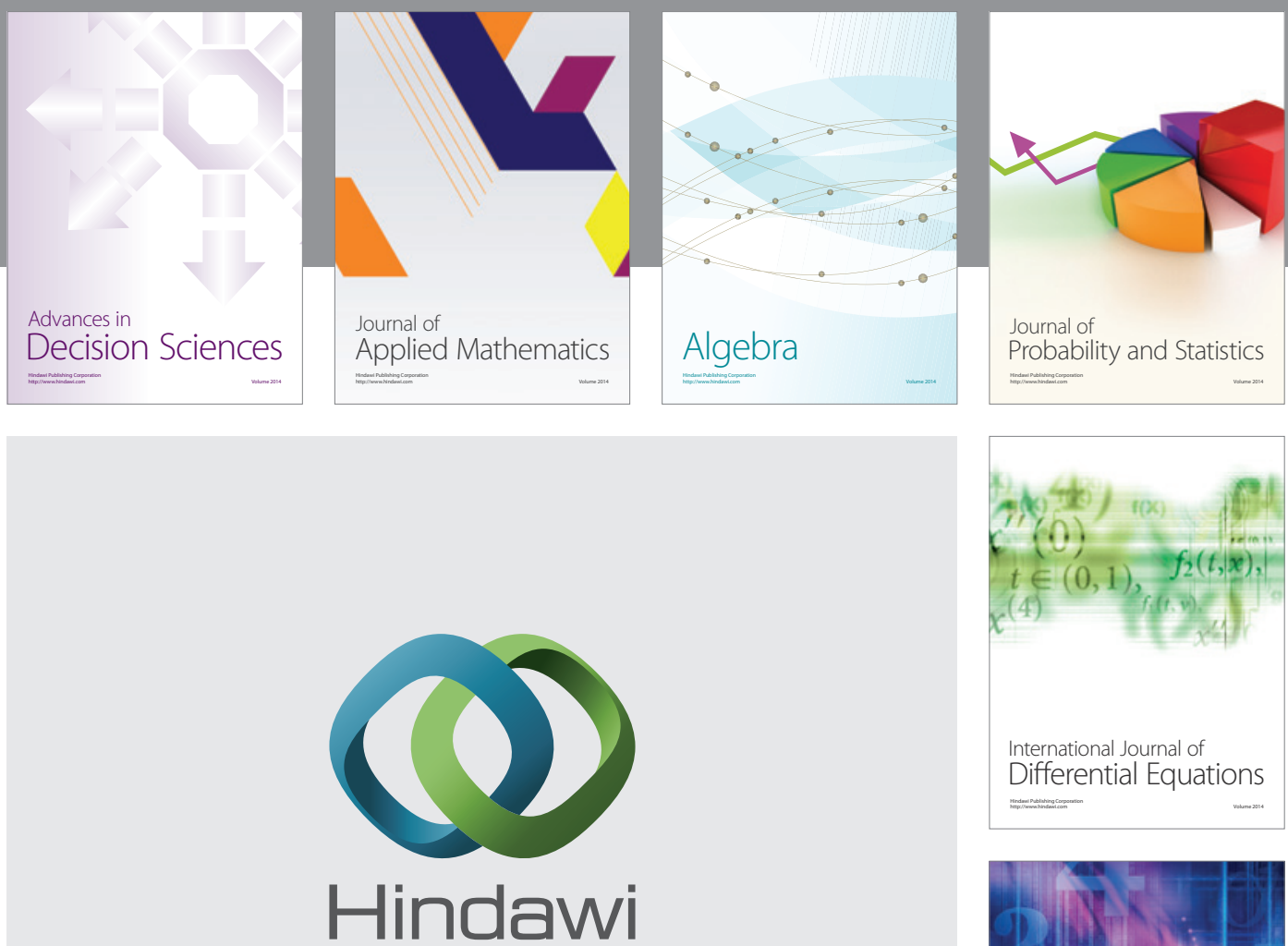

Submit your manuscripts at http://www.hindawi.com
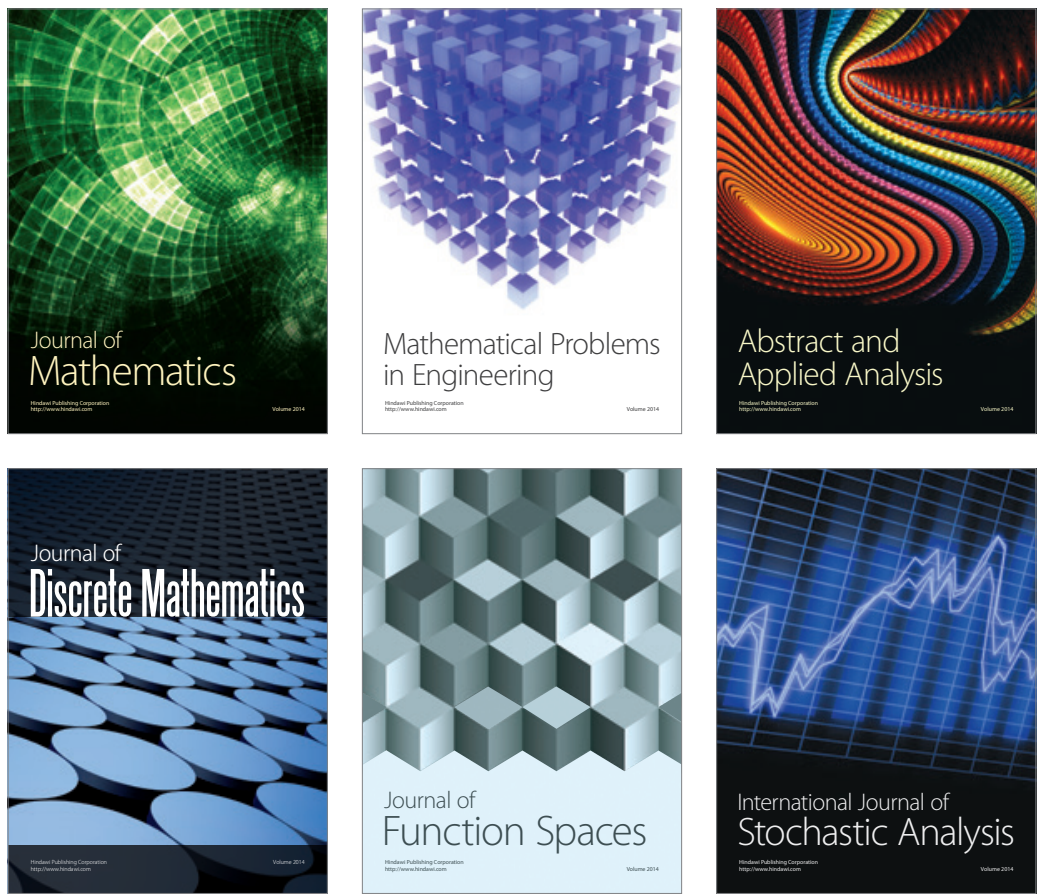

Journal of

Function Spaces

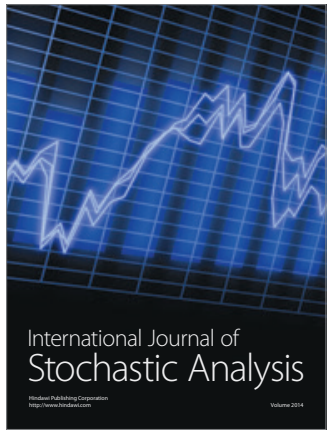

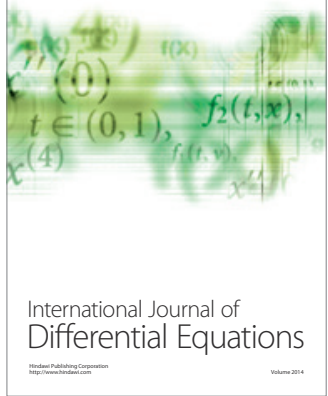
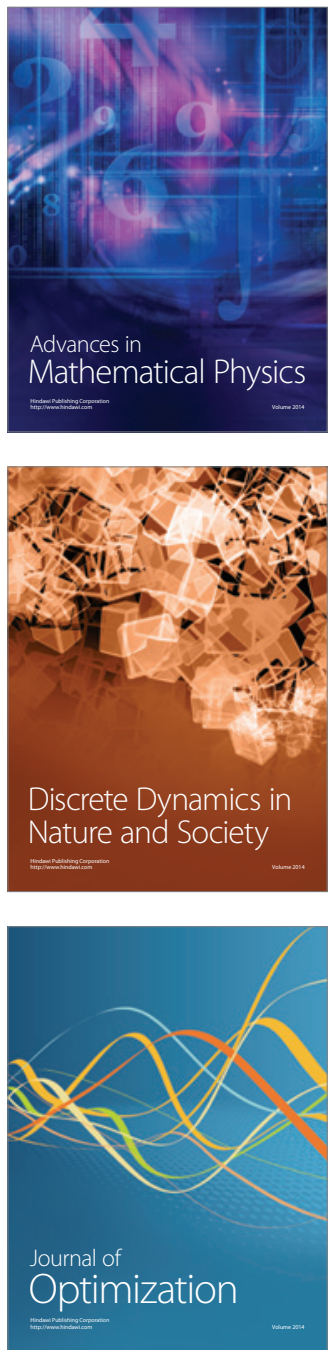